\title{
OPTIMIZACIÓN DEL PROCESO DE ACIDULACIÓN DE SOAPSTOCK DE ACEITE DE SOYA
}

\section{Optimization of the Acidulation Process of Soapstock of Soybean Oil}

\author{
Luis Carlos Valdés Luna \\ Universidad del Atlántico, Programa de Ingeniería Quimica, Grupo de Investigación \\ Gestión de la Calidad. Puerto Colombia, Colombia.
}

Recibido: 07/04/2018 • Aprobado: 19/0572018

\section{RESUMEN}

Este artículo presenta el estudio que tiene como propósito determinar las condiciones de operación adecuadas del proceso batch de acidulación del subproducto de la refinación química del aceite de soya -también denominado soapstock- con el objetivo de optimizar el tiempo de reacción; para lo cual se implementó un diseño estadístico experimental Box-Behnken, con tres niveles y tres factores. Las variables de proceso estudiadas fueron: temperatura de proceso $\left(80,0-100,0^{\circ} \mathrm{C}\right)$, velocidad del agitador $(300-500 \mathrm{rpm})$ y dosificación de ácido sulfúrico en la reacción $(6,0-10,0 \%)$. La variable de respuesta estudiada fue el tiempo de reacción del proceso. Se propuso un modelo predictivo de segundo orden para esta variable, obteniendo las superficies de respuesta y las condiciones favorables que minimizan el tiempo de reacción a 13 minutos por lote; las cuales fueron de $100^{\circ} \mathrm{C}$, agitación de $300 \mathrm{rpm}$ y 7,68\% de ácido sulfúrico. Los resultados indicaron que la temperatura, la velocidad del agitador y la dosificación de ácido sulfúrico tienen una influencia significativa sobre el tiempo de reacción resultante.

Palabras clave: proceso de acidulación, soapstock, aceite de soya, superficie de respuesta.

\section{Abstract}

The purpose of this study is to determine the adequate operating conditions of the batch process of by product of acidification the chemical refining of soybean oil-also known as soapstock-with the aim of optimizing the reaction time. An experimental Box-Behnken statistical design was implemented, with three levels and three factors. The process variables studied were: agitator speed $(300-500 \mathrm{rpm})$, process temperature $(80,0-100,0$ $\left.{ }^{\circ} C\right)$ and dosage of sulfuric acid in the reaction $(6,0-10,0 \%)$. The response variable studied was the reaction time of the process. A second-order predictive model was proposed for this variable, obtaining response surfaces and favorable conditions that minimize the reaction time to 13 minutes per batch which were $100^{\circ} \mathrm{C}$, agitation

lcvaldesl@gmail.com, orcid.org/0000-0002-2222-4326 
of $300 \mathrm{rpm}$ and $7.68 \%$ of sulfuric acid. The results indicated that the temperature, the agitator speed and the dosage of sulfuric acid, have a significant influence on the resulting reaction time.

Key words: acidulation process, soapstock, soybean oil, response surface.

\section{INTRODUCCIÓN}

Los constituyentes principales de los aceites crudos de origen vegetal, son los triacilglicéridos, pero también contienen otros componentes en cantidades menores, los cuales afectan significativamente sus propiedades químicas y físicas. Estos componentes menores son jabones, fosfolípidos, tocoferoles, esteroles, resinas, carbohidratos, proteínas, traza de metales y pigmentos como el gosipol, el caroteno y la clorofila (Woerfel, 1995). La mayor parte de ellos son indeseados, ya que afectan el aspecto, el olor, el sabor y el desempeño del aceite, y por consiguiente aportan una experiencia sensorial poco satisfactoria en su consumo. Por ello, se hace necesario someter el aceite vegetal crudo a un proceso de refinación que permita eliminar estas impurezas (Gupta, 2017).

El soapstock de aceites de oleaginosas es el subproducto primordial de la industria de refino de aceites vegetales (Down, 1998). Este subproducto suele considerarse un excedente de inventario sin utilidad inmediata, pero es de gran valor cuando se logra recuperar y procesar de manera correcta (Woerfel, 1983; PilotoRodriguez, 2014).

Los jabones que contiene el soapstock son las sales de sodio de los ácidos grasos neutralizados; la eficiencia del desdoblamiento del soapstock depende en gran medida del contenido de fosfolípidos que posea el aceite procesado, ya que, si se encuentra muy elevado con relación a los jabones, se promueve la formación de emulsiones en el proceso de desdoblamiento, las cuales son difíciles de romper, afectando seriamente la separación del aceite y el agua ácida (Dorsa, 2009). El método más usual es garantizar el desgomado con ácido en una etapa previa a la neutralización para mantener los jabones separados de los fosfolípidos. Otro método tendiente a disminuir la generación de emulsiones de los fosfolípidos en el soapstock, con buenos resultados, es el método de post-saponificación del soapstock, en el cual el soapstock es tratado agregando soda cáustica y agitando durante 20 minutos a alta temperatura (Gupta, 2017).

La acidulación se realiza en un tanque de reacción mezclando el soapstock con ácido sulfúrico concentrado. El ácido sulfúrico es usado comúnmente para este proceso, aunque el ácido clorhídrico también se utiliza para estos fines. El ácido fuerte se dosifica por medio de un sistema automático de control del $\mathrm{pH}$ del tanque, requiriendo un $\mathrm{pH}$ entre 2,5 a 3,5 y temperaturas cercanas a los $90^{\circ} \mathrm{C}$. El ácido se mezcla con el soapstock en virtud del agitador axial, o en algunos procesos, con barbotaje de vapor (Dorsa, 2009).

Al completarse la reacción, se obtiene un aceite ácido en fase sobrenadante, y una fase acuosa que contiene sulfato de sodio (o cloruro de sodio si se usó ácido clorhídrico en la reacción), y otros compuestos hidrosolubles. El acidulado de soya, también llamado aceite ácido de soya (fase sobrenadante) es secado al vacío, y luego es vendido a la industria alimenticia como ingrediente para concentrado de animales (Escalera-Valente, 2016; Реña, 2014). La fase acuosa residual tiene alto contenido de demanda bioquímica de oxígeno (DBO) de 40.000 a 90.000 , y bajo $\mathrm{pH}$. Sin embargo, cuando se trata correctamente para adaptar 
el $\mathrm{pH}$ y remover los materiales no miscibles se biodegrada fácilmente (Watson \& Meierhoefer, 1976). La reglamentación para los vertimientos contiene nuevos criterios para DBO, DQO, sólidos totales, grasas y aceites (Torrez et al., 2017). Esta fase acuosa residual es neutralizada con solución cáustica antes de ser reutilizada (Jollez et al., 2001; Garro et al., 2003). Se ha sugerido el tratamiento del agua ácida con una lechada de cal para neutralizar a un $\mathrm{pH}$ de 7 a 7,5 (Woerfel, 1995).

El objetivo del presente estudio es desarrollar un modelo empírico para el estudio del tiempo de reacción de la acidulación y determinar las condiciones de operación óptimas para las variables del proceso, contribuyendo a la disminución de los tiempos en la operación y la eficiencia del proceso.

\section{Materiales y métodos}

Los materiales utilizados fueron el soapstock de aceite de soya, obtenido de un proceso de refinación química convencional (GEA Westfalia). En la tabla 1 , se puede observar la caracterización realizada a la muestra representativa del soapstock sometido al experimento. Para la reacción se utilizó ácido sulfúrico concentrado industrial 98\%.

Para la determinación de materia insaponificable, fósforo, aceite neutro, ácidos grasos totales y $\mathrm{pH}$ del soapstock y la acidez libre oleica del acidulado se usaron los métodos AOCS (1998).

Para la determinación de la humedad se utilizó un medidor de humedad absoluta digital PCE Instruments, referencia PCE-MA 110.

\subsection{Proceso de acidulación y diseño de experimento}

Las muestras de soapstock fueron sometidas a un pretratamiento de saponificación, adicionando $0,30 \%$ de soda cáustica y agitando la mezcla durante $20 \mathrm{mi}-$ nutos, regulando la temperatura a $90^{\circ} \mathrm{C}$.
El proceso de acidulación consistió en adicionar al soapstock un ácido inorgánico fuerte, a alta temperatura y presión atmosférica, hasta total conversión de los jabones en ácidos grasos. En este estudio se determinó el comportamiento del tiempo de reacción y la acidez libre del acidulado de soya en función de tres variables de proceso controlables. El experimento se realizó en lotes de $50 \mathrm{~g}$, variando la temperatura, la dosificación de ácido sulfúrico y la velocidad del agitador.

El proceso de acidulación es tipo batch, con agitación axial, garantizando un mezclado constante de la muestra; por las características apreciables de la viscosidad de la mezcla se sugiere utilizar un mezclador axial de cuchillas (Gupta, 2017).

Se utilizó un diseño estadístico Box-Behnken de 3 variables de proceso, con 3 repeticiones del punto central, resultando en 15 corridas. Los factores controlables estudiados fueron: temperatura de proceso $\left(80,0-100,0{ }^{\circ} \mathrm{C}\right)$, velocidad del agitador $(300-500$ $\mathrm{rpm})$ y dosificación de ácido sulfúrico en la reacción $(6,0-10,0 \%)$. Los factores estudiados y sus niveles de variación para el diseño experimental se encuentran en la Tabla 2.

\section{TABLA 1.}

Caracterización de la muestra de soapstock

\begin{tabular}{|l|c|}
\hline Parámetro & Resultado \\
\hline Insaponificable (\%) & $4,15 \pm 0,20$ \\
\hline Fósforo (ppm) & $95 \pm 1$ \\
\hline Aceite neutro (\%) & $10,50 \pm 0,50$ \\
\hline Ácidos Grasos Totales (\%) & $35,50 \pm 1,00$ \\
\hline Humedad (\%) & $57,05 \pm 1,05$ \\
\hline pH & $8,90 \pm 0,10$ \\
\hline
\end{tabular}

Fuente: elaborado por el autor.

Al terminar el proceso de acidulación, cada muestra se dejó en reposo con el fin de separar por decantación 
natural la fase acuosa. El punto final de acidulación se definió visualmente, cuando las dos fases presentaban una adecuada separación, el sobrenadante un tono café oscuro, con un $\mathrm{pH}$ inferior a 3 .

\section{TABla 2.}

Esquema del diseño experimental del proceso de acidulación del soapstock de soya

\begin{tabular}{|l|c|c|c|c|}
\hline Factores & Bajo & Intermedio & Alto & Unidades \\
\hline Temperatura & 80 & 90 & 100 & ${ }^{\circ} \mathrm{C}$ \\
\hline Velocidad del Agitador & 300 & 400 & 500 & $\mathrm{rpm}$ \\
\hline Dosis de Ácido Sulfúrico & 6,0 & 8,0 & 10,0 & $\% \mathrm{p} / \mathrm{p}$ \\
\hline
\end{tabular}

Fuente: elaborado por el autor.

TABla 3.

Diseño de Box-Behnken para el análisis de superficie. Trece experimentos en total con tres puntos centrales.

\begin{tabular}{|c|c|c|c|}
\hline Temperatura, ${ }^{\circ} \mathrm{C}$ & Velocidad del agitador, rpm & Ácido sulfúrico, \%p/p & Tiempo de reacción (min) \\
\hline 80 & 300 & 8,0 & 28 \\
\hline 90 & 500 & 10,0 & 17 \\
\hline 80 & 500 & 8,0 & 29 \\
\hline 100 & 400 & 6,0 & 21 \\
\hline 90 & 400 & 8,0 & 18 \\
\hline 90 & 400 & 8,0 & 19 \\
\hline 80 & 400 & 6,0 & 48 \\
\hline 80 & 400 & 10,0 & 27 \\
\hline 100 & 300 & 8,0 & 15 \\
\hline 100 & 500 & 8,0 & 23 \\
\hline 90 & 300 & 6,0 & 27 \\
\hline 90 & 300 & 10,0 & 25 \\
\hline 90 & 400 & 8,0 & 19 \\
\hline 90 & 500 & 6,0 & 34 \\
\hline 100 & 400 & 10,0 & 15 \\
\hline
\end{tabular}

Fuente: elaborado por el autor.

Los resultados obtenidos se analizaron por medio de la metodología de superficie de respuesta (MSR), utilizando análisis de regresión múltiple con ajuste a modelos matemáticos de segundo orden, conteniendo términos lineales, cuadráticos e interacciones entre variables independientes (ver Ecuación No 1). 


$$
Y=\beta_{0}+\sum \beta_{i} x_{i}+\sum \beta_{i i} x_{i i}^{2}+\sum \beta_{i j} x_{i} x_{j}+\epsilon
$$

Ecuación No 1

Estos análisis estadísticos se hicieron de acuerdo a lo propuesto por Box \& Behnken (Box, et al., 1978) y por análisis de varianza (ANOVA). Las superficies de respuesta se graficaron para validar el efecto de las variables sobre las respuestas. Los resultados fueron analizados considerando el coeficiente de determinación $\mathrm{R}^{2}$ y el diagrama de Pareto.

\section{RESUlTADOS Y DisCUSIÓN}

La Tabla No 4 muestra los efectos estimados, los coeficientes del modelo ajustado y la desviación estándar para cada coeficiente.

Se observó que todos los factores y las interacciones
TT, TA, TD, AA, AD y DD, son significativas con una confiabilidad del 95\%, lo cual implica un $\mathrm{p}<0,05$. Por otra parte, el modelo ajustado presentó un coeficiente de correlación favorable de 93,17\%, es decir, el modelo explica el 93,17\% de la variabilidad del tiempo de reacción, con una carencia de ajuste no significativa con valor $\mathrm{p}$ inferior al 0,05 .

En la Figura 1, se muestra el diagrama de Pareto donde se presentan los efectos estandarizados con $\mathrm{p}=$ 0,05. Se puede observar que los factores A, C y CC son los más significativos y los que más influencia tienen sobre la variable de respuesta. El valor negativo de los coeficientes de las variables temperatura y dosis de ácido sulfúrico, indica que el tiempo de reacción fue favorecido con valores altos de estas variables. Resultados similares donde la temperatura y la dosificación de ácido son las variables de mayor significancia han sido reportados en estudios precedentes (Woerfel, 1983; Mag, Green \& Kwong, 1983.

TABla 4.

Efectos estimados y coeficientes ajustados para tiempo de reacción en función de temperatura, velocidad del agitador y dosis de ácido sulfúrico

\begin{tabular}{|c|c|c|c|c|}
\hline Término & Efecto & Coeficiente & Error estándar & Valor - P \\
\hline Constante & & 641,125 & \\
\hline T: Temperatura & $-14,50$ & $-9,3$ & 2,66771 & 0,0029 \\
\hline A: Agitación & 2,00 & $-0,120833$ & 2,66771 & 0,4872 \\
\hline D: Ac. Sulfúrico & $-11,50$ & $-34,4167$ & 2,66771 & 0,0076 \\
\hline TT & 7,08 & 0,0354167 & 3,92676 & 0,1311 \\
\hline TA & 3,50 & 0,00175 & 3,77271 & 0,3961 \\
\hline TD & 7,50 & 0,1875 & 3,77271 & 0,1035 \\
\hline AA & 3,08 & 0,000154167 & 3,92676 & 0,4679 \\
\hline AD & $-7,50$ & $-0,01875$ & 3,77271 & 0,1035 \\
\hline DD & 11,08 & 1,38542 & 3,92676 & 0,0370 \\
\hline
\end{tabular}

$\mathrm{R}^{2}=93,17 \% \cdot \mathrm{R}^{2}$ (ajustado) $=80,86 \%$

Fuente: elaborado por el autor. 


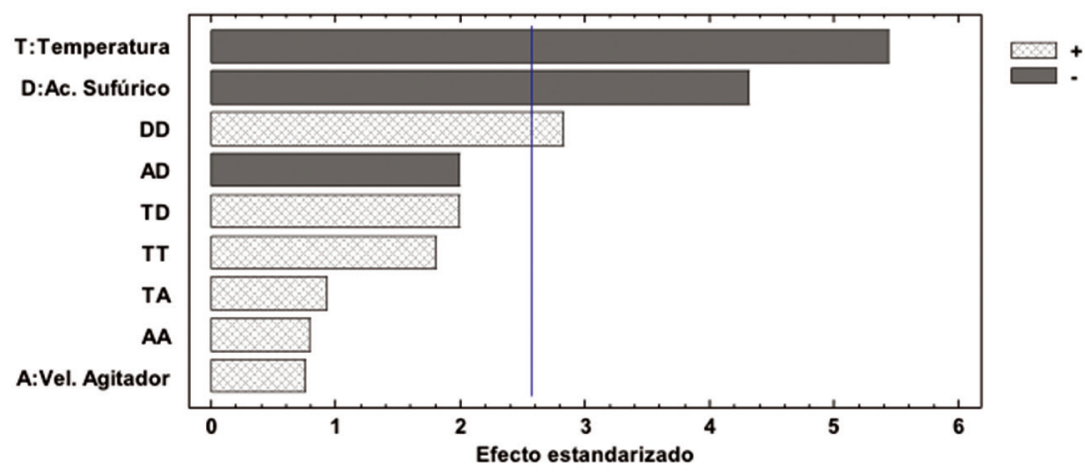

Fig. 1. Diagrama de Pareto de los efectos estandarizados con $\mathrm{p}=0,05$.

Fuente: elaborado por el autor.

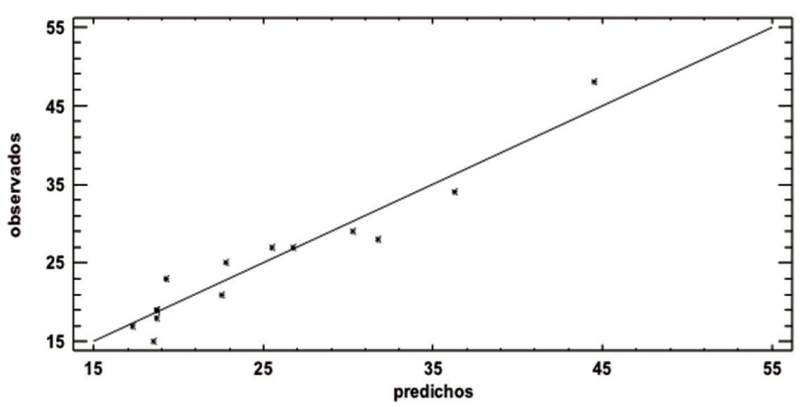

Fig. 2. Gráfico de correlación de respuesta predicha versus los resultados observados

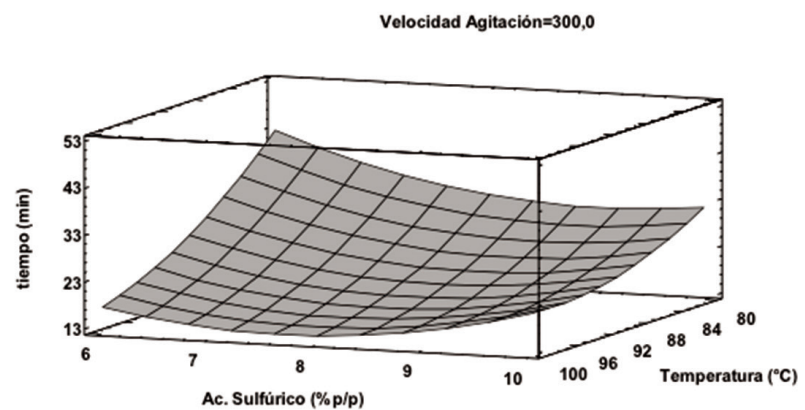

Fig. 3 (a). Superficie de respuesta

Fuente: elaborado por el autor.

\section{Velocidad Agitación=300,0}

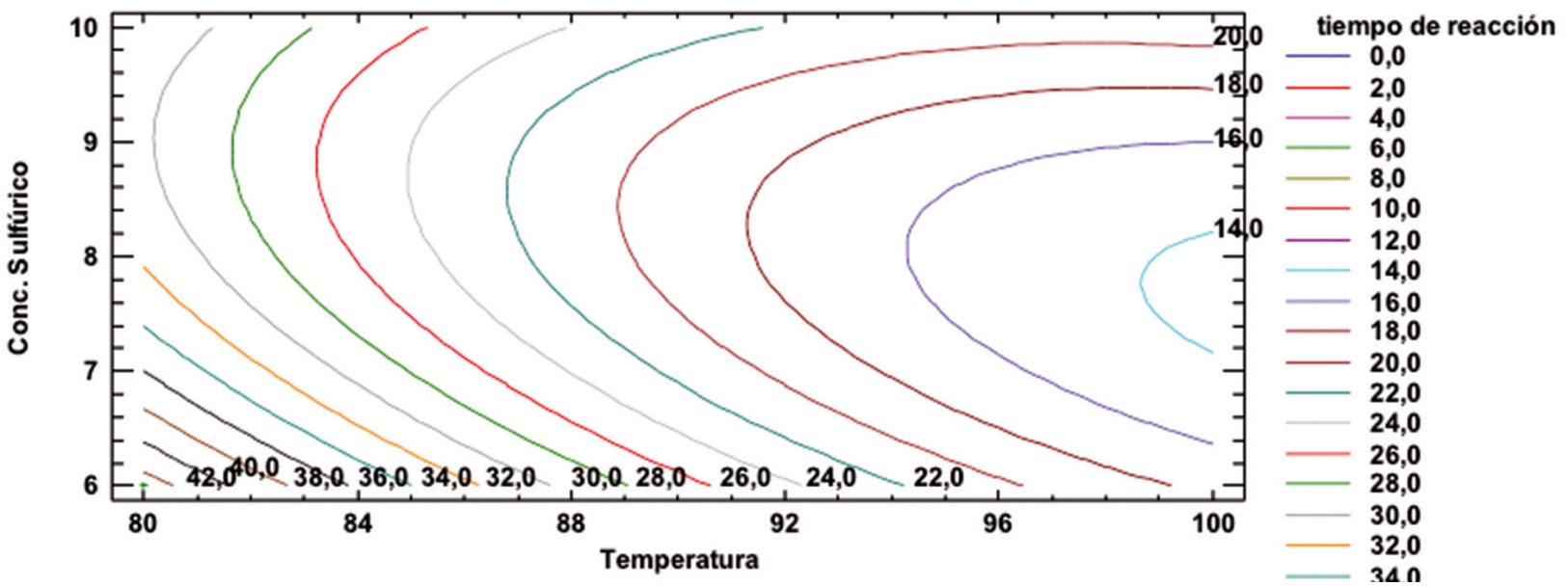

Fig. 3b. Superficie de respuesta para el tiempo de reacción de la acidulación, con velocidad del agitador constate de 300 rpm. a. Superficie de respuesta, b. Contorno de la superficie de respuesta

Fuente: elaborado por el autor. 
La Figura 3 muestra la superficie de respuesta para el tiempo de reacción en función de la cantidad de ácido sulfúrico dosificado en la reacción y la temperatura de reacción. Se puede observar una zona óptima que minimiza el tiempo de reacción cercana a los $100^{\circ} \mathrm{C}$ y dosificaciones entre 7,0\% y 9,0\% de ácido sulfúrico. En valores altos de temperatura de reacción se acelera el proceso de desdoblamiento de soapstock (Truong, et al., 2017).

El modelo obtenido presenta una curvatura causada en mayor medida por el aporte significativo del efecto cuadrático de la dosificación de ácido sulfúrico, CC. El tiempo de reacción empieza a ascender drásticamente cuando la temperatura se encuentra en valores inferiores a los $85^{\circ} \mathrm{C}$ y dosis de ácido sulfúrico inferiores al $7 \% \mathrm{p} / \mathrm{p}$.

Las fuentes de variabilidad se asocian al control de la temperatura de la reacción, ya que el carácter exotérmico de la misma provoca oscilaciones repentinas de la temperatura. Por otra parte, el tipo de agitador utilizado puede alterar el fenómeno de transferencia másica entre las especies reaccionantes, siendo esta una fuente de perturbación.

También, las características físicas y químicas del soapstock utilizado determinan el comportamiento de la reacción, siendo la humedad del soapstock y la concentración de grasa neutra y fosfolípidos lo que determina la viscosidad de la mezcla, así como la formación de emulsiones. Esta se relaciona linealmente con la concentración de jabones, la cual deriva de la concentración de ácidos grasos libres totales del aceite (Yooritphun, et al., 2016).

Cuando ha finalizado la reacción, existen dos métodos alternativos para separar las fases resultantes: centrifugación o un recipiente de decantación. Para este estudio, se empleó un recipiente de decantación, de donde se separó la fase sobrenadante sin mayor dificultad. La eficiencia de este proceso está muy influenciada por el contenido de gomas (fosfolípidos) en el jabón que produce un alto grado de emulsificación. Para contenidos altos de fosfolípidos solo la centrifugación es el método eficaz (Laoretani, 2017) (Lehtinen, 2017).

En procesos similares, como la esterificación enzimática de acidulado, la humedad del soapstock interfiere con la actividad de las enzimas, para estos casos, la determinación de la humedad en la materia prima se debe considerar relevante y debe ser añadida como factor controlable en el diseño (Cruz et al., 2017).

El tiempo de reacción del acidulado en el experimento desarrollado se ajusta a la ecuación (1). Si se utiliza este modelo, se puede alcanzar un tiempo de reacción mínimo de 13,6 minutos con las variables de proceso establecidas en $100^{\circ} \mathrm{C}$ de temperatura de reacción, velocidad del agitador en 300 rpm y 7,68\% de ácido sulfúrico en la mezcla reaccionante.

$$
\begin{aligned}
& \boldsymbol{Y}(\min )=641,125-9,3 \boldsymbol{T}-0,120833 \boldsymbol{A}-34,4167 \boldsymbol{D} \\
& +0,0354167 \boldsymbol{T}^{2}+0,00175 \boldsymbol{T}+0,1875 \boldsymbol{T} \boldsymbol{D}+ \\
& 0,000154167 \boldsymbol{A}^{2}-0,01875 A \boldsymbol{D}+1,38542^{*} \boldsymbol{D}^{2}
\end{aligned}
$$

Donde $Y$ es la respuesta pronosticada del tiempo de reacción (en minutos), $T$ es la temperatura de reacción $\left({ }^{\circ} \mathrm{C}\right), A$ es la velocidad del agitador (rpm) y $D$ es la dosificación de ácido sulfúrico en la reacción (\%p/p).

En este modelo no se considera la interacción triple de los factores controlables, por lo que esta variabilidad hace parte del error. Los diseños de Box-Behnken presentan las principales ventajas de descubrir las posibles interacciones entre los parámetros y también ahorrar tiempo al reducir el número de experimentos (Yu, 2017).

El modelo anterior es válido para temperaturas en el intervalo $80-100^{\circ} \mathrm{C}$, velocidades del agitador en el intervalo de 300-500 rpm y dosificaciones de ácido sulfúrico en el intervalo de $6-10 \% \mathrm{p} / \mathrm{p}$. Una característica de la estadística multivariada es la simplicidad de sus respuestas: esto significa que los modelos matemáticos usados para interpretar los datos pueden ser complejos y estructurados, pero las respuestas que se otorgan deben ser claras (Zuluaga, 2011). 


\section{Conclusiones}

Por medio del diseño de superficie Box-Behnken, se pudo minimizar el tiempo de reacción, sugiriendo 13 minutos como valor mínimo, con las variables de proceso ajustadas en $100^{\circ} \mathrm{C}, 7,68 \%$ de ácido y agitación en $300 \mathrm{rpm}$.

El modelo estadístico permite predecir el tiempo de reacción del proceso y es una alternativa empírica para la comprensión de este proceso. Además, el uso del diseño de superficie Box-Behnken permitió determinar la interacción entre las variables del proceso y sus efectos en el tiempo de reacción, reduciendo el número de experimentos a realizar.

\section{RECONOCIMIENTOS}

El autor agradece el apoyo de la Universidad del Atlántico, la Facultad de Ingeniería y al Grupo de Investigación de Gestión de la Calidad, por el fomento del uso de Control Estadístico de Procesos para el mejoramiento de los procesos productivos.

El autor agradece a los editores y evaluadores por su contribución a la publicación de este artículo.

\section{REFERENCIAS}

AOCS. (1998). Official Methods and Recommended Practices of the American Oil Chemists Society. Champaign: AOCS.

Box, G. E.P., Hunter, W. G. \& Hunter, J. S. (1978). Statistics for experiments - An introduction to design, data analysis and model building. New York: Wiley \& Sons.

Cruz, M., Cardinal Pinho, S., Mota, R., Fonseca Almeida, M. \& Maia Dias, J. (2017). Enzymatic esterification of acid oil from soapstocks obtained in vegetable oil refining: Effect of enzyme concentration. Renewable Energy, (124), 165-171. http:// dx.doi.org/10.1016/j.renene.2017.06.053

Escalera-Valente, F., Loya-Olguín, J. L., Carmona-Gasca, C. A., Martínez-González, S. \& Avila-Ramos, F. (2016). Effect of oregano oil supplementation in diets formed using either crude soybean oil or acidulated soybean oil soapstock as source of energy on the growth performance parameters of broilers. $A r$ chivos de Medicina Veterinaria, 48(2), 209-214. Recuperado en: http://mingaonline.uach.cl/pdf/amv/v48n2/art11.pdf

Dorsa, R. (2009). Desdoblamiento de las borras de neutralización. Revista A\&G, (75), 264-268.

Down, M. K. (1998). Gas chromatographic characterization of soapstocks from vegetable oil refining. J. Chromatography, (816), 185-193.

Garro, J. M. Lemieux, A., Jollez, P. \& Cadoret, N. (2003). Method for fractionating greases trap waste and uses of fractions therefrom. Patente WO Patent 03/038020.

Gupta, M. K. (2017). Batch Acidulation Process. En: Practical Guide to Vegetable Oil Processing, (pp. 119-124). Lynnwood: AOCS Press. Elsevier Inc.

Jollez, P., Garro, J. M., Estaque, L., Lemieux, A. \& Cadoret, N. (2001). Production of high grade and high concentration of free fatty acids from residual oils, fats and greases. Patente WO Patent $01 / 83655$.

Laoretani, D. S., Fisher, C. D. \& Iribarren, O. A. (2017). Selection among alternative processes for the disposal of soapstock. Food and Bioproducts Processing. (101), 177-183. https://doi. org/10.1016/j.fbp.2016.10.015

Lehtinen, O., Nugoho, R. W. N., Lehtimaa, T., Vierros, S., Hiekkataipale, P., Ruokolainen, J., Sammalkorpi, M. \& Österberg, M. (2017). Effect of temperature, water content and free fatty acid on reverse micelle formation of phospholipids in vegetable oil. Colloids and Surfaces B: Biointerfaces, 160(1), 355-363. https://doi.org/10.1016/j.colsurfb.2017.09.050

Mag, T. K., Green, D. H. \& Kwong, A. T. (1983). Continuous Acidulation of Soapstock and Recovery of Acid Oil. JAOCS, 60(5), https://doi.org/10.1007/BF02660217

Peña, J. E. M., Vieira, S. L., Borsatti, L., Pontin, C. \& Rios, H. V. (2014). Energy utilization of by-products from the soybean oil industry by broiler chickens: acidulated soapstock, lecithin, glycerol and their mixture. Brazilian Journal of Poultry Science, 16(4), http://dx.doi.org/10.1590/1516-635X1604437-442

Piloto-Rodriguez, R., Melo, E. A., Goyos-Pérez, L. \& Verhelst, S. (2014). Conversion of by-products from the vegetable oil industry into biodiesel and its use in internal combustion engines: a review. Brazilian Journal of Chemical Engineering, 31(2). http://dx.doi.org/10.1590/01046632.20140312 s00002763

Scavariello, E. (2004). Optimización del proceso de acidulación de la borra de neutralización de aceite de salvado de arroz. Grasas y Aceites, 55, (2), 155-159.

Torres, J. A., Contento Rubio, O. F. \& Herrera Orozco, I. (2017). Análisis de ciclo de vida para una biorefinería derivada de residuos agrícolas de palma aceitera (Elaeis guineensis). Revista Publicaciones e Investigación, 11(1), 13-36. 
Truong, H. T., Van, M. D., Huynh, L. D., Nguyen, L. T., Tuan, A. D., Thanh, T. L. X., Phuoc, H. D. Takenaka, N., Imamura, K. \& Maeda, Y. (2017).

A Method for Ferulic Acid Production from Rice Bran Oil Soapstock Using a Homogenous System. Appl. Sci., 7(8), 796, 2017. https://doi.org/10.3390/app7080796

Watson, K. S. \& Meierhoefer, C. H. (1976). Use or disposal of byproducts and spent material from the vegetable oil processing industry in the U.S. JAOCS, (53), https://doi.org/10.1007/ BF02605740

Woerfel, J. B. (1995). Soybean oil processing byproducts and their utilization, En: Practical Handbook of Soybean Processing and Utilization, (pp. 297-313). AOCS Press, Champaign. https:// doi.org/10.1016/B978-0-935315-63-9.50021-8
Woerfel, J. B. (1983). Alternatives for processing of soapstock. J. Am. Oil Chem. Soc., (60), 262A-265A, https://doi.org/10.1007/ BF02543509

Xiao-Lan, Y. \& He, Y. (2017). Application of box-behnken designs in parameters optimization of differential pulse anodic stripping voltammetry for lead (II) determination in two electrolytes. Scientific Reports (Nature Publisher Group), (7), 1-8. https://doi.org/10.1038/s41598-017-03030-2

Yooritphun, K., Lilitchan, S., Aryusuk, K. \& Krisnangkura, K. (2017). Effect of Selected Polyhydric Alcohols on Refining Oil Loss in the Neutralization Step. J Am Oil Chem Soc, (94), 301308. https://doi.org/10.1007/s11746-016-2924-4

Zuluaga, C. M. (2011). Análisis estadístico multivariado como herramienta Estratégica para el Control de procesos de calidad en la industria agroalimentaria. Revista Publicaciones e Investigación. 5(1), 143-157, https://doi.org/10.22490/25394088.587 
